\title{
RASSF1A and SIRT6 in non-small cell lung cancer: Relationship with clinical outcome
}

\author{
TAO CHEN, ZHAOJUN SUN, FENGLING LIU and QIANG WANG
}

The Second Department of Surgery, Chinese Medicine Hospital in Linyi City, Linyi, Shandong 276002, P.R. China

Received December 23, 2016; Accepted April 26, 2017

DOI: 10.3892/ol.2017.6172

\begin{abstract}
This study investigated the expression of RASSF1A and SIRT6 in non-small cell lung cancer (NSCLC) and its relationship with clinical prognosis. The expression in 122 cases of NSCLC tissues (NSCLC group) and 122 cases of normal lung tissues (NOR group) during the same period were detected by immunohistochemical Super Pic Ture ${ }^{\mathrm{TM}}$ Polymer two-step method, and the relationship between its expression and the clinicopathological features and prognosis of patients was analyzed. The positive expression rates of RASSF1A and SIRT6 in NSCLC group were lower than those in the normal group (55.74 vs. $84.43 \%$ and 52.46 vs. $82.49 \%, \mathrm{P}<0.01)$. The differences in expression intensity of RASSF1A in NSCLC tissues between different tumor pathological types, tumor differentiation degrees and lymph node metastases were statistically significant, and the differences in expression intensity of SIRT6 between different TNM stages, tumor differentiation degrees and lymph node metastases were statistically significant. There was a positive correlation between the expression of RASSF1A and SIRT6 in NSCLC group ( $\mathrm{r}=0.532, \mathrm{P}<0.01$ ). The 3-year survival rate of patients with high-expression of RASSF1A was higher than in those with low-expression of RASSF1A (81.33 vs. $39.45 \%$, log-rank $\left.\chi^{2}=19,102, \mathrm{P}<0.01\right)$; the 3 -year survival rate of patients with high-expression of SIRT6 was higher than in those with low-expression of SIRT6 (83.51 vs. $42.43 \%$, log-rank $\chi^{2}=17,180, \mathrm{P}<0.01$ ). The low expression of RASSF1A and SIRT6 and lymph node metastasis were the risk factors affecting the prognosis of NSCLC patients. There is a better correlation between the expression of RASSF1A and SIRT6 in NSCLC tissues, and the detection of their expression is of great significance in the judgement of clinicopathological features and prognosis of NSCLC patients.
\end{abstract}

Correspondence to: Dr Qiang Wang, The Second Department of Surgery, Chinese Medicine Hospital in Linyi City, 211 Jiefang Road, Lanshan, Linyi, Shandong 276002, P.R. China

E-mail:wmuquw@163.com

Key words: non-small cell lung cancer, RASSF1A, SIRT6, clinical outcome

\section{Introduction}

At present, lung cancer has the highest incidence among all types of cancers around the world, which is also the leading cause of death of cancer patients (1). Non-small cell lung cancer (NSCLC) accounts for the majority of lung cancers. The prognosis of patients with lung cancer is still poor, and the 5-year survival rate after diagnosis is $<16 \%$ (2). According to NCCN guidelines, the postoperative 5-year survival rate of Stage-I NSCLC patients for whom the radiotherapy and chemotherapy were not recommended after operation is only about $61.3 \%$, and there are still a considerable number of patients who die within 5 years due to the local tumor recurrence or distant metastasis (3). Douillard et al conducted the large-scale clinical study and found that the postoperative adjuvant chemotherapy was helpful for the improvement of survival rate of some lung cancer patients with a high risk of recurrence and metastasis (4). Therefore, screening the high-risk patients through evaluating the related factors to the prognosis of NSCLC patients, and providing adjuvant therapy in time are of great clinical importance in improving the 5-year survival rate of patients with early-stage NSCLC.

The new type of candidate tumor suppressor gene, Ras association domain family gene 1A (RASSF1A), is a kind of newly-discovered gene with speculated tumor-suppressing function, located in $3 \mathrm{p} 21.3$. This gene is expressed in the normal tissues, but there is a higher rate of expression loss in lung cancer and other tumors. It is found in the study that the lung cancer cell line A549 without the expression of RASSF1A after being transfected with this gene can reduce the cell clonal formation, inhibit the anchorage-independent growth, slow down the growth rate of transplanted tumor inoculated to nude mice and significantly reduce the pulmonary metastasis capacity of A549 cells $(5,6)$.

As a key member in long-lived proteins, SIRT6 is closely related to the tumor formation and progression, which is also significantly associated with the survival rate of cancer patients. Studies have shown that SIRT6 shows a significant anticancer effect in lung cancer, especially in NSCLC. In NSCLC tissue and cell lines, mRNA and protein expression levels in SIRT6 are generally decreased (7). Further studies have shown that SIRT6 can inhibit the proliferation of NSCLC cells by inhibiting Twist1 (7).

This study aimed to detect the expressions of RASSF1A and SIRT6 in NSCLC tissues and normal lung tissues by 
immunohistochemical method, and investigated its relationship with clinicopathological features and prognosis of NSCLC patients.

\section{Materials and methods}

Patients and sample source. In total 122 NSCLC patients (NSCLC group) in the Chinese Medicine Hospital of Linyi City from January to December 2011 were selected, including 89 males and 33 females aged 33-76 years with an average age of $(59.83 \pm 8.94)$ years; none of the cases received radiotherapy or chemotherapy before operation, which was confirmed pathologically. According to the TNM staging criteria revised by UICC (Union for International Cancer Control) in 2009 , there were 48 cases in Stage I, 33 cases in Stage II and 41 cases in Stage III. According to the WHO classification standards of lung cancer, there were 65 cases of squamous carcinoma, 42 cases of adenocarcinoma and 15 cases of adenosquamous carcinoma. There were 76 cases of high and moderate differentiation and 46 cases of low differentiation, and there were 62 cases of lymph node metastasis and 60 cases of non-lymph node metastasis. Another 122 cases of normal lung tissues (normal group) without infiltration of cancer cells confirmed by $H \& E$ staining were collected from the same patients. The follow-up was from February 1, 2011 to January 31, 2016, and the survival period was calculated from the date of surgery to the expiration date of follow-up, or to the date of death due to recurrence and metastasis. The application of samples was approved by the Ethics Committee of the Chinese Medicine Hospital of Linyi City and signed written informed consent was obtained from patients or their families.

Methods. The samples were fixed with $10 \%$ neutral formalin immediately after surgical excision, followed by conventional paraffin embedding and serial section. The section thickness was $5 \mu \mathrm{m}$. Conventional immunohistochemical Super Pic Ture $^{\mathrm{TM}}$ Polymer (Biosharp, Hefei, China) two-step method was used for detection according to the instructions. The high-pressure high-temperature thermal remediation was used for antigen retrieval. Rabbit polyclonal RASSF1A antibody (dilution, 1:100; cat. no. ab180801) and rabbit polyclonal SIRT6 antibody (dilution, 1:100; cat. no. ab135566) and secondary goat anti-rabbit (HRP) IgG antibody (dilution, 1:2,000; cat. no. ab6721) were all purchased from Abcam (Cambridge, MA, USA). Diaminobenzidine (DAB) chromogenic kit was purchased from Fuzhou Maixin Biotech. Co., Ltd., (Fuzhou, China). The known positive sections were used as the positive control and PBS as the negative control, instead of the primary antibody.

Judgement of results. RASSF1A protein was mainly expressed on the membrane structure of cells. Positive SIRT6 was mainly expressed on the cell membrane and partially in the cytoplasm. The assessment and analysis were conducted according to the percentage of staining cells and staining degree: 10 high-power fields (x400) were randomly observed for each case, and 100 cells were counted in each field; the positive cell rate $=$ positive cell count/observed cell count $x 100 \%$. Scoring standards of positive cell rate: $\leq 10 \%$ for 0 point, $11-25 \%$ for 1 point, $26-50 \%$ for 2 points, $51-75 \%$
Table I. Comparison of positive expressions of RASSF1A and SIRT6 between two groups.

\begin{tabular}{lccr}
\hline Group & $\mathrm{n}$ & RASSF1A $(\%)$ & SIRT6 $(\%)$ \\
\hline NSCLC & 122 & $68(55.74)$ & $64(52.46)$ \\
NOR & 122 & $103(84.43)$ & $101(82.49)$ \\
$\chi^{2}$ & & 23,746 & 25,537 \\
\hline
\end{tabular}

NSCLC, non-small cell lung cancer.

for 3 points, and $>75 \%$ for 4 points. Staining degree: yellow, 1 point; brown yellow, 2 points; and dark brown 3 points; the scores of positive cell rate and staining degree were multiplied to obtain the final score: 0 point for negative (-), 1-4 points for weakly positive $(+), 5-8$ points for moderately positive $(++)$ and 9-12 points for strongly positive $(+++)$. In the analysis, $(-)$ and $(+)$ were recognized as the low expression, and $(++)$ and $(+++)$ were recognized as the high expression.

Statistical analysis. SPSS 18.0 software (SPSS, Inc., Chicago, IL, USA) was used for data processing. The $\chi^{2}$ test was used for intergroup comparison of enumeration data. Spearman rank correlation was used for the correlation analysis. Kaplan-Meier analysis was used for survival data, the survival curves were drawn, and the differences were detected by logrank test. COX risk model was used for analysis of prognostic factors. $\mathrm{P}<0.05$ suggested that the difference was statistically significant.

\section{Results}

Comparison of the positive expression of RASSF1A and SIRT6 between the two groups. The positive expression rates of RASSF1A and SIRT6 in NSCLC group were lower than those in the normal group $(\mathrm{P}<0.01)$ (Table I). In both groups, RASSF1A was mainly expressed on membrane structure (cell or nuclear membrane) and SIRT6 was expressed on the cell membrane; the positive expression of RASSF1A and SIRT6 was high expressioned in the normal group (Fig. 1).

Relationship between expressions of RASSF1A and SIRT6 and clinicopathological features of patients in NSCLC group. The differences in expression intensity of RASSF1A and SIRT6 had no statistical significance between genders, ages, smoking habits, tumor sites and maximum tumor diameters. The differences in expression intensity of RASSF1A in NSCLC tissues had statistical significance under different pathological patterns of the tumor, tumor differentiation degrees and lymph node metastases. The differences in expression intensity of SIRT6 in NSCLC tissues had statistical significance under the tumor differentiation degrees, TNM stages and lymph node metastases (Table II).

Analysis of correlation between expression of RASSF1A and SIRT6 in NSCLC group. There was a positive correlation between expression of RASSF1A and SIRT6 in NSCLC group ( $\mathrm{r}=0.532, \mathrm{P}<0.01)$ (Table III). 
Table II. Comparison of the expressions of RASSF1A and SIRT6 between different clinicopathological characteristic groups of NSCLC.

\begin{tabular}{|c|c|c|c|c|c|}
\hline \multirow{2}{*}{$\begin{array}{l}\text { Pathological } \\
\text { characteristic }\end{array}$} & \multirow[b]{2}{*}{$\mathrm{n}$} & \multicolumn{2}{|c|}{ RASSF1A } & \multicolumn{2}{|l|}{ SIRT6 } \\
\hline & & Low expression (\%) & $\chi^{2}$ & Low expression (\%) & $\chi^{2}$ \\
\hline Sex & & & 0.121 & & 0.537 \\
\hline Male & 89 & $45(50.56)$ & & $54(60.67)$ & \\
\hline Female & 33 & $17(51.51)$ & & $18(54.55)$ & \\
\hline Age (years) & & & 0.345 & & 0.592 \\
\hline$\leq 60$ & 57 & $31(54.38)$ & & $35(61.40)$ & \\
\hline$>60$ & 65 & $32(49.23)$ & & $36(55.38)$ & \\
\hline Smoking & & & 0.297 & & 0.103 \\
\hline Yes & 93 & $48(51.61)$ & & $55(59.14)$ & \\
\hline No & 29 & $16(55.17)$ & & $17(58.62)$ & \\
\hline Tumor site & & & 0.134 & & 0.212 \\
\hline Left & 49 & $25(51.02)$ & & $27(55.10)$ & \\
\hline Right & 73 & 37 (50.68) & & $42(57.53)$ & \\
\hline Tumor size $(\mathrm{cm})$ & & & 0.023 & & 0.247 \\
\hline$\leq 3$ & 55 & $28(50.90)$ & & $30(54.55)$ & \\
\hline$>3$ & 67 & $34(50.75)$ & & $35(52.24)$ & \\
\hline Pathological type & & & 9.862 & & 0.751 \\
\hline Adenocarcinoma & 42 & $13(30.95)$ & & $22(52.38)$ & \\
\hline Squamous carcinoma & 65 & $38(58.46)$ & & $39(60.00)$ & \\
\hline Adenosquamous carcinoma & 15 & $11(73.33)$ & & $10(66.67)$ & \\
\hline Differentiation & & & 6.138 & & 4.619 \\
\hline High-medium & 76 & $30(39.47)$ & & $36(47.37)$ & \\
\hline Low & 46 & $32(69.57)$ & & $34(73.91)$ & \\
\hline TNM & & & 2.125 & & 8.176 \\
\hline Stage I & 48 & $22(45.83)$ & & $22(45.83)$ & \\
\hline Stage II-III & 74 & $42(56.76)$ & & $49(66.22)$ & \\
\hline Lymph node metastasis & & & 5.739 & & 5.177 \\
\hline Yes & 62 & $37(59.68)$ & & $42(67.74)$ & \\
\hline No & 60 & $24(40.00)$ & & $26(43.33)$ & \\
\hline
\end{tabular}

NSCLC, non-small cell lung cancer.

Table III. Correlation of the expression of RASSF1A and SIRT6 in NSCLC.

\begin{tabular}{lccr}
\hline & \multicolumn{3}{c}{ SIRT6 } \\
\cline { 2 - 4 } RASSF1A & Low expression & High expression & Total \\
\hline Low expression & 55 & 12 & 67 \\
High expression & 18 & 37 & 55 \\
Total & 73 & 49 & 122 \\
\hline
\end{tabular}

$\mathrm{r}=0.532, \mathrm{P}<0.01$. NSCLC, non-small cell lung cancer.

The relationship between the expression of RASSF $1 A$ and SIRT6 and the prognosis of patients with NSCLC. A total of 122 NSCLC patients were followed up for 1-60 months with the median time of 44 months, and 3 cases were lost to follow-up. The 3-year survival rates of patients with high-expression RASSF1A and low-expression RASSF1A were 83.82\% (57/68) and $40.74 \%$ (22/54), respectively; and the difference had statistical significance (log-rank $\left.\chi^{2}=18,992, \mathrm{P}<0.01\right)$ (Fig. 2A). The 3-year survival rates of patients with high-expression of SIRT6 and low-expression of SIRT6 were $85.71 \%$ (42/49) and $45.20 \%$ (33/73), respectively; and the difference had statistical significance (log-rank $\left.\chi^{2}=16,680, \mathrm{P}<0.01\right)$ (Fig. 2B). The 3 -year survival rates of patients with high-expression RASSF1A and high-expression of SIRT6, and patients with low-expression of RASSF1A and low-expression of SIRT6 were $89.19 \%(33 / 37)$ and $34.55 \%$ (19/55), respectively; and the difference had statistical significance $\left(\log -\right.$ rank $\left.\chi^{2}=24,385, \mathrm{P}<0.01\right)$ (Fig. $\left.2 \mathrm{C}\right)$.

Multivariate COX regression model analysis. COX proportional hazard regression model was used for analysis with the 

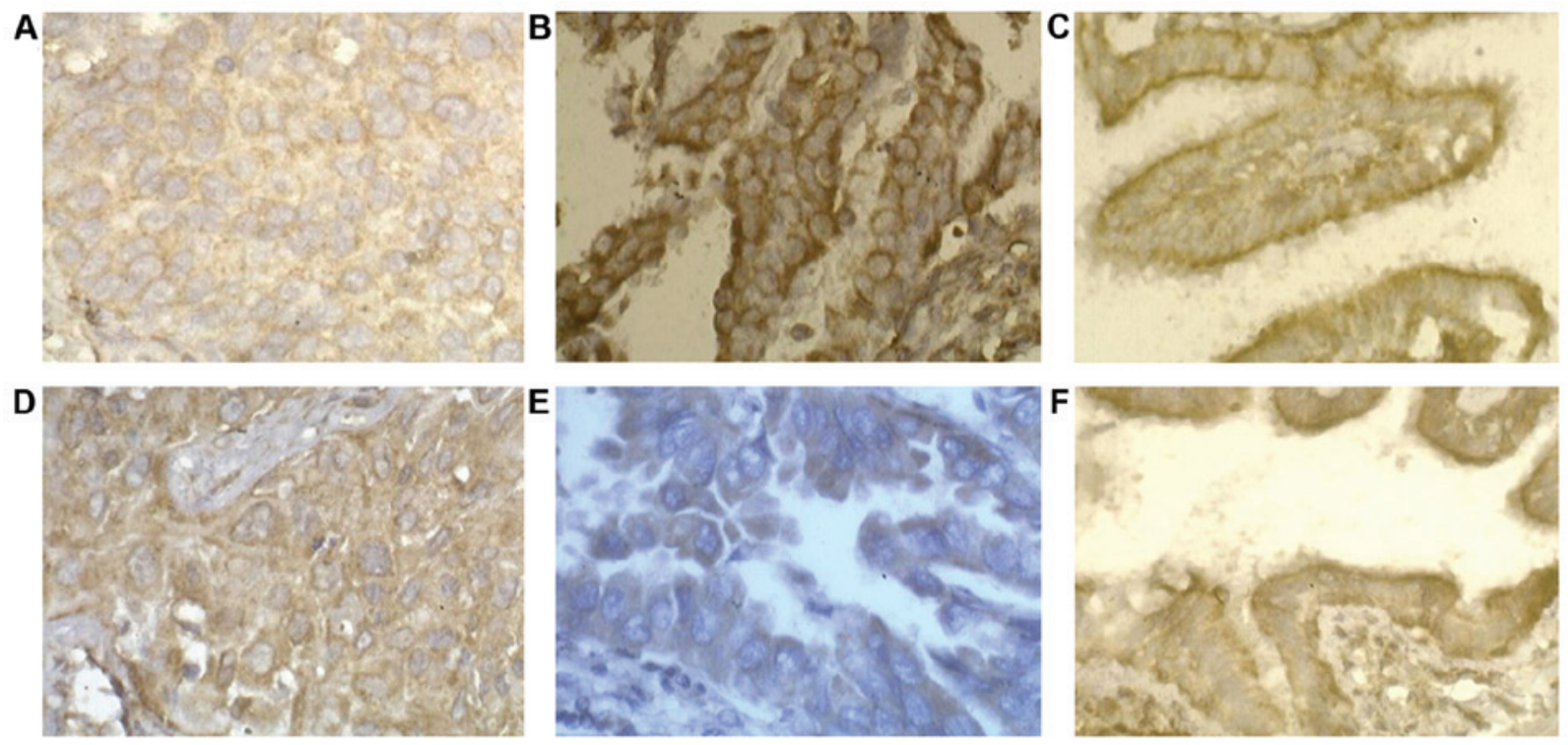

Figure 1. The expression of RASSF1A and SIRT6 in different lung tissues. (A) The expression of RASSF1A in squamous carcinoma. (B) The expression of RASSF1A in adenocarcinoma. (C) The expression of RASSF1A in normal lung tissue. (D) The expression of SIRT6 in squamous carcinoma. (E) The expression of SIRT6 in adenocarcinoma. (F) The expression of SIRT6 in normal lung tissue.

A

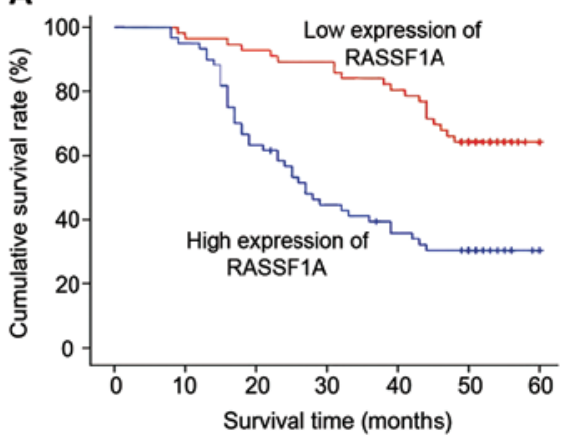

B

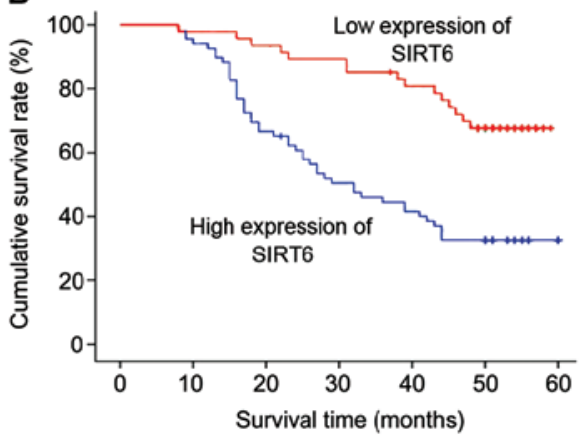

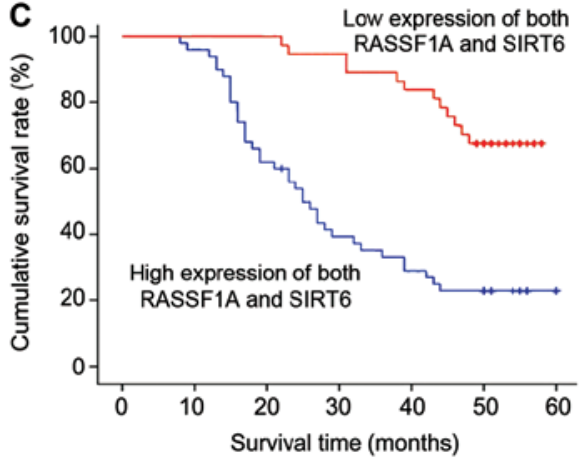

Figure 2. The survival function of different groups. (A) The correlation between the expression of RASSF1A and the survival time of patients with NSCLC. (B) The correlation between the expression of SIRT6 and the survival time of patients with NSCLC. (C) The correlation between the high expression of both RASSF1A and SIRT6, the low expression of both RASSF1A and SIRT6 and the survival time of patients with NSCLC. NSCLC, non-small cell lung cancer.

Table IV. COX proportional hazards model for various factors related to prognosis in patients with NSCLC.

\begin{tabular}{lrrrrrr}
\hline Variable & $\beta$ & SE & Wald $\chi^{2}$ & P-value & RR & RR 95\% CI \\
\hline Age & -0.131 & 0.252 & 0.147 & 0.673 & 0.859 & $0.541-1.515$ \\
Sex & 0.014 & 0.364 & 2.005 & 0.172 & 1.716 & $0.883-3.248$ \\
Differentiation & 0.459 & 0.372 & 0.018 & 0.947 & 0.978 & $0.495-1.897$ \\
Lymph node metastasis & 1.411 & 0.327 & 20.523 & $<0.001$ & 4.012 & $2.372-7.586$ \\
Pathological type & -0.119 & 0.245 & 0.566 & 0.517 & 0.914 & $0.613-1.317$ \\
Tumor size & -0.034 & 0.236 & 0.047 & 0.796 & 0.885 & $0.512-1.526$ \\
Common low expression & 1.314 & 0.362 & 19.021 & $<0.001$ & 3.872 & $1.971-6.315$ \\
\hline
\end{tabular}

NSCLC, non-small cell lung cancer.

gender (male=1, female=2), age, pathological pattern, tumor differentiation degree, maximum tumor diameter, whether there was lymph node metastasis (yes $=1, \mathrm{no}=2$ ) and whether RASSF1A and SIRT6 were lowly expressed (yes $=1$, no $=2$ ) as independent variables and the survival time as dependent variable. The results showed that the low expressions of RASSF1A and SIRT6 and lymph node metastasis were the risk factors affecting the prognosis of NSCLC patients (Table IV). 


\section{Discussion}

RASSF1A is a relatively unique molecule in the RAS family. The expression of RASSF1A is usually reduced in the development process of malignant tumors (8). The in vitro studies have also shown that RASSF1A can inhibit the proliferation, survival, invasion and metastasis of tumor cells (9-11). Chen et al knocked out the RASSF1A genes in non-tumor cells in bronchus via RNA interference, and found that the decreased expression of RASSF1A could promote cell migration and invasion (12). Huang et al found that the expression quantity of RASSF1A in lung cancer was significantly decreased with the increase of lung cancer infiltration (13), and the expression loss of RASSF1A was significantly associated with the high stage, high infiltration, poor differentiation and high proliferation index of lung cancer, and the proliferation and growth of A549 cells with RASSF1A overexpression were significantly decreased (14-16). Pastuszak-Lewandoska et al studied and showed that 64 out of 112 cases of primary NSCLC had no expression or low expression of RASSF1A, which was consistent with the results of this study (17).

In this study, RASSF1A was highly expressed in normal lung tissues and significantly decreased in NSCLC tissues, and its expression intensity was different under different pathological patterns of tumor, tumor differentiation degrees and lymph node metastases (17). The positive expression of SIRT6 has the function of inhibiting tumor metastasis (18); the decreased expression or expression loss of SIRT6 and the decreased cell adhesion cause the infiltrative growth of cells to the peripheral region $(7,19,20)$; once the necessary conditions for metastasis are obtained, cells can get out of the primary lesion, followed by invasion and metastasis.

In recent years, the studies on lung cancer and breast cancer, papillary thyroid carcinoma, bladder, prostate, colorectal and nasopharyngeal cancer have confirmed that the decreased expression of SIRT6 was significantly correlated with the tumor differentiation, invasion and metastasis (21-25). In this study, SIRT6 expression was significantly decreased in most of the NSCLC tissues, and its expression intensity was different in different TNM stages, tumor differentiation degrees and lymph node metastases. The results of this study showed that the high or low expressions of RASSF1A and SIRT6 occur easily in patients with adenosquamous carcinoma, poor differentiation, high stage and lymph node metastasis, and the low expressions of RASSF1A and SIRT6 are the risk factors of poor prognosis of NSCLC patients. The expression of RASSF1A and SIRT6 in NSCLC tissues have a better correlation and consistency, suggesting that RASSF1A and SIRT6 play similar roles in the signal transduction pathway promoting the occurrence and infiltration of the tumor.

The low or no expression of RASSF1A in lung cancer may be mainly caused by the low acetylation of histones, and the significantly low expression of RASSF1A can be seen in lung cancer cell lines treated with histone deacetylase inhibitors. The low expression of RASSF1A can promote the migration and infiltration of bronchial epithelial cells, which activates the phosphatidylinositol 3-kinase (PI3K)-Akt signaling pathway mainly through the activation of serine/threonine protein kinase (Akt). Thaler et al found that RASSF1A deletion could promote tumorigenesis and tumor cell growth, and promote the occurrence of Akt signaling pathway in breast cancer cells (26). Romano et al proposed that RASSF1A plays an important role in the migration and budding of endothelial cells and the formation of blood capillaries, and its partial mechanism is the inhibition of Ras/ROCK signaling pathway (27).

Thus, it can be seen that the expression loss of RASSF1A may be one of the features of lung cancer cell infiltration, and its mechanism may be related to the activation of PI3K/Akt signaling pathway. Studies have also found that SIRT6 has an effect on tumor cell proliferation through mediating the activation effect of epidermal growth factor receptor on PI3K/Akt (28). SIRT6 inhibits the proliferation and infiltration of NSCLC cells, which is realized possibly by downregulating the expression of Raf/MEK/ERK. When Raf genes are knocked out, the proliferation and infiltration capabilities of NSCLC cells are significantly decreased. It was observed that RASSF1A and SIRT6, to a certain extent, have the same effects in the signal transduction pathway of tumor occurrence and development.

\section{References}

1. Leong D, Rai R, Nguyen B, Lee A and Yip D: Advances in adjuvant systemic therapy for non-small-cell lung cancer. World J Clin Oncol 5: 633-645, 2014.

2. Jing W, Li N, Wang Y, Liu X, Liao S, Chai H and Tu J: The prognostic significance of long noncoding RNAs in non-small cell lung cancer: A meta-analysis. Oncotarget 8: 3957-3968, 2016

3. Ettinger DS, Wood DE, Akerley W, Bazhenova LA, Borghaei H, Camidge DR, Cheney RT, Chirieac LR, D'Amico TA, Dilling TJ, et al: NCCN guidelines insights: Non-small cell lung cancer, version 4.2016. J Natl Compr Canc Netw 14: 255-264, 2016.

4. Douillard JY, Lehur PA, Vignoud J, Blottière H, Maurel C, Thedrez P, Kremer M and Le Mevel B: Monoclonal antibodies specific immunotherapy of gastrointestinal tumors. Hybridoma 5 (Suppl 1): S139-S149, 1986.

5. Dallol A, Agathanggelou A, Tommasi S, Pfeifer GP, Maher ER and Latif F: Involvement of the RASSF1A tumor suppressor gene in controlling cell migration. Cancer Res 65: 7653-7659, 2005.

6. Deng ZH, Zhou JH, Cao HQ, Sheng M and Weng JF: Proliferation inhibition of human lung adenocarcinoma cell line A549 transfected by RASSF1A gene. J Cent South Univ (Med Sci) 30: 193-196, 2005

7. Han Z, Liu L, Liu Y and Li S: Sirtuin SIRT6 suppresses cell proliferation through inhibition of Twist 1 expression in non-small cell lung cancer. Int J Clin Exp Pathol 7: 4774-4781, 2014.

8. Fukatsu A, Ishiguro F, Tanaka I, Kudo T, Nakagawa K, Shinjo K, Kondo Y, Fujii M, Hasegawa Y, Tomizawa K, et al: RASSF3 downregulation increases malignant phenotypes of non-small cell lung cancer. Lung Cancer 83: 23-29, 2014.

9. Avruch J, Praskova M, Ortiz-Vega S, Liu M and Zhang XF: Nore1 and RASSF1 regulation of cell proliferation and of the MST1/2 kinases. Methods Enzymol 407: 290-310, 2006.

10. Dubois F, Keller M, Calvayrac O, Soncin F, Hoa L, Hergovich A, Parrini MC, Mazières J, Vaisse-Lesteven $\mathrm{M}$, Camonis $\mathrm{J}$, et al: RASSF1A suppresses the invasion and metastatic potential of human non-small cell lung cancer cells by inhibiting YAP activation through the GEF-H1/RhoB pathway. Cancer Res 76: 1627-1640, 2016.

11. Whang YM, Park KH, Jung HY, Jo UH and Kim YH: Microtubule-damaging agents enhance RASSF1A-induced cell death in lung cancer cell lines. Cancer 115: 1253-1266, 2009.

12. Chen K, Li BK, Xu K, Xu A, Liu C, Zheng S, Xu Y, Jia C, Liu Q, Li H, et al: Effect of stable DNA methyltransferase $3 \mathrm{~b}$ knockdown on proliferation and apoptosis in bladder cancer cells in vitro. Nan Fang Yi Ke Da Xue Xue Bao 35: 1524-1529, 2015 (In Chinese).

13. Huang YZ, Wu W, Wu K, Xu XN and Tang WR: Association of RASSF1A promoter methylation with lung cancer risk: A meta-analysis. Asian Pac J Cancer Prev 15: 10325-10328, 2014. 
14. Fu L and Zhang S: RASSF1A promotes apoptosis and suppresses the proliferation of ovarian cancer cells. Int J Mol Med 33: 1153-1160, 2014

15. Ko E, Lee BB, Kim Y, Lee EJ, Cho EY, Han J, Shim YM, Park J and Kim DH: Association of RASSF1A and p63 with poor recurrence-free survival in node-negative stage I-II non-small cell lung cancer. Clin Cancer Res 19: 1204-1212, 2013.

16. Dittfeld C, Richter AM, Steinmann K, Klagge-Ulonska A and Dammann RH: The SARAH domain of RASSF1A and its tumor suppressor function. Mol Biol Int 2012: 196715, 2012.

17. Pastuszak-Lewandoska D, Kordiak J, Migdalska-Sek M, CzarneckaKH, Antczak A, Górski P, Nawrot E, KiszałkiewiczJM, Domańska D and Brzeziańska-Lasota E: Quantitative analysis of mRNA expression levels and DNA methylation profiles of three neighboring genes: FUS1, NPRL2/G21 and RASSF1A in non-small cell lung cancer patients. Respir Res 16: 76, 2015.

18. Bai L, Lin G, Sun L, Liu Y, Huang X, Cao C, Guo Y and Xie C: Upregulation of SIRT6 predicts poor prognosis and promotes metastasis of non-small cell lung cancer via the ERK1/2/MMP9 pathway. Oncotarget 7: 40377-40386, 2016.

19. Azuma Y, Yokobori T, Mogi A, Altan B, Yajima T, Kosaka T, Onozato R, Yamaki E, Asao T, Nishiyama M, et al: SIRT6 expression is associated with poor prognosis and chemosensitivity in patients with non-small cell lung cancer. J Surg Oncol 112: 231-237, 2015

20. Cai Y, Sheng ZY and Liang SX: Radiosensitization effect of overexpression of adenovirus-mediated SIRT6 on A549 non-small cell lung cancer cells. Asian Pac J Cancer Prev 15: 7297-7301, 2014.

21. Bae JS, Park SH, Jamiyandorj U, Kim KM, Noh SJ, Kim JR Park HJ, Kwon KS, Jung SH, Park HS, et al: CK2 $\alpha /$ CSNK2A1 phosphorylates SIRT6 and is involved in the progression of breast carcinoma and predicts shorter survival of diagnosed patients. Am J Pathol 186: 3297-3315, 2016.
22. Bhardwaj A and Das S: SIRT6 deacetylates PKM2 to suppress its nuclear localization and oncogenic functions. Proc Natl Acad Sci USA 113: E538-E547, 2016.

23. Demir IE, Ceyhan GO and Friess H: Epigenomic therapies: The potential of targeting SIRT6 for the treatment of pancreatic cancer. Expert Opin Ther Targets 21: 1-3, 2017.

24. Lee N, Ryu HG, Kwon JH, Kim DK, Kim SR, Wang HJ, Kim KT and Choi KY: SIRT6 depletion suppresses tumor growth by promoting cellular senescence induced by DNA damage in HCC. PLoS One 11: e0165835, 2016.

25. Wolf K and Strand S: Assessing the histone deacetylase activity of SIRT6 in primary murine hepatocytes via proximity ligation assay. Methods Mol Biol 1510: 149-158, 2017.

26. Thaler S, Hähnel PS, Schad A, Dammann R and Schuler M: RASSF1A mediates p $21^{\text {Cipl/Waf1 }}$-dependent cell cycle arrest and senescence through modulation of the Raf-MEK-ERK pathway and inhibition of Akt. Cancer Res 69: 1748-1757, 2009.

27. Romano D, Matallanas D, Weitsman G, Preisinger C, Ng T and Kolch W: Proapoptotic kinase MST2 coordinates signaling crosstalk between RASSF1A, Raf-1, and Akt. Cancer Res 70: 1195-1203, 2010

28. Sundaresan NR, Vasudevan P, Zhong L, Kim G, Samant S, Parekh V, Pillai VB, Ravindra PV, Gupta M, Jeevanandam V, et al: The sirtuin SIRT6 blocks IGF-Akt signaling and development of cardiac hypertrophy by targeting c-Jun. Nat Med 18: 1643-1650, 2012 